\title{
Generative Capacities of Cellular Automata Codification for Evolution of NN Codification
}

\author{
Germán Gutiérrez, Inés M. Galván, José M. Molina, and Araceli Sanchis \\ SCALAB. Departamento de Informática. Universidad Carlos III de Madrid. \\ Avda. Universidad 30, 28911-Leganés (Madrid)-SPAIN. \\ \{ggutierr,igalvan, masm\}@inf.uc3m.es, molina@ia.uc3m.es
}

\begin{abstract}
Automatic methods for designing artificial neural nets are desired to avoid $\mathrm{t}$ he $\mathrm{l}$ aborious an $\mathrm{d}$ er ratically hu man ex pert's $\mathrm{j}$ ob. Evolutionary computation $\mathrm{h}$ as be en us ed a s a searc $\mathrm{h} t$ echnique $\mathrm{t}$ o fi nd appropriate NN architectures. Direct and indirect en coding methods are us ed to codify the net architecture i nto the chr omosome. A reform ulation of a $\mathrm{n}$ i ndirect enc oding method, bas ed on t wo bi-dimensional cel lular aut omata, a nd i ts generative capacity are presented.
\end{abstract}

\section{Introduction}

The arch itecture d esign i s a fu ndamental s tep in t he successful application of Artificial Neural Networks (ANN), and it is unfortunately still a h uman ex perts job. Most of the methods are based on evolutionary computation paradigms, Evolutionary Artificial Neural Networks (EANN). A wide review of using evolutionary techniques to evolve different aspects of neural networks can be find in (Yao, 1999).

The interest of this paper is focused on the design of Feedforward Neural Networks (FNN) arch itectures us ing genetic algo rithms. Th ere are two mai $\mathrm{n}$ representation approaches fo $\mathrm{r}$ cod ification of FN N in the ch romosome to find the optimal FNN architecture. On e, b ased on $\mathrm{t}$ he $\mathrm{c}$ omplete re presentation of all the possible connections, direct encoding, relatively simple and straightforward to implement. But large architectures, for complex tasks, requires much larger chromosomes ( Miller et al., 89; Fogel, 1990; Alba et al., 1993). Other based on an indirect representation of the architecture, indirect encoding schemes. Those schemes consists of codifying, not the complete $n$ etwork, but a co mpact $r$ epresentation of it, av oiding the scalability problem and reducing the length of the genotype. (Kitano, 1990; Gruau, 1992;Molina et al., 2000).

In this work, an indirect constructive encoding scheme, based on cellular automata (Wolfram, 1998), is reformulated. Tw o bi-dimensional cellular a utomata are us ed to generate FNN architectures proposed. It is inspired on the idea that only a few s eeds for the initial configuration of cellular automata can produce a wide variety of FNN architectures, generative capacity. And this generative ca pacity, the search s pace of $\mathrm{NN}$ covered by cellular encoding, is shown too. 


\section{Description of Cellular System}

The g lobal s ystem is c omposed of th ree differen $t$ modules: the Gen etic Algorithm Module, the Ce llular M odule a nd t he $\mathrm{N}$ eural $\mathrm{N}$ etwork $\mathrm{M}$ odule ( Fig 1). A $11 \mathrm{t}$ he modules are related to make a general procedure. The cellular module is composed of two bi -dimensional cellular systems a nd t akes c harge of ge nerating $\mathrm{F} N \mathrm{~N}$ architectures. Initial configurations of cellular systems are given by several seeds and the rules of the systems are applied to generate final configurations, which correspond to a FNN architecture. The generated FNN is trained and relevant information about the goodness of FNN is used as the fitness value for the genetic module. The genetic algorithm module takes charge of generating the positions of the seeds (codified in the chromosome) in the two-dimensional grid of cellular systems, which determine initial configurations of cellular systems. In [Gutierrez et. al., 2001] a detailed description of Neural Network and Genetic Algorithm Modules c an be found. In the ne xt section, the new formulation of the Cellular Module is presented.

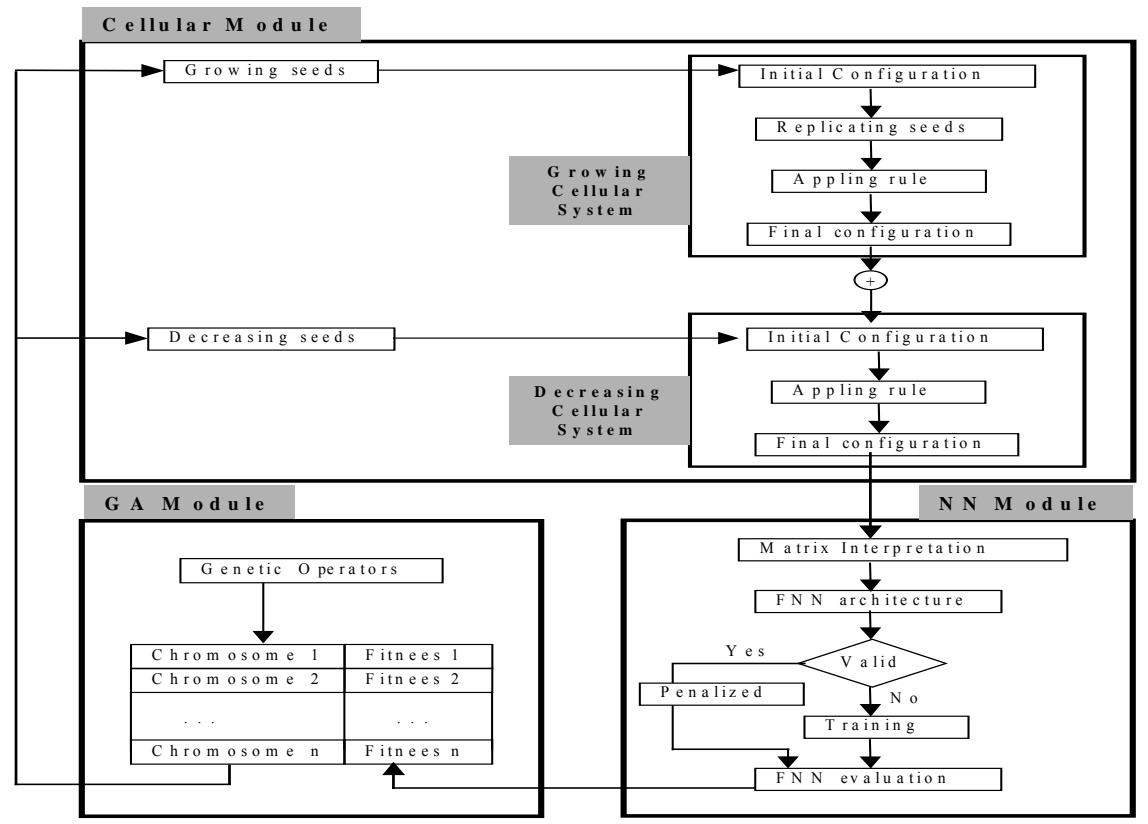

Fig. 1. System's architecture and modules relationship.

\section{Cellular Module}

The cellular module (Fig 1) is composed b y t wo bi-dimensional cell ular s ystem. "Growing cellular s ystem" a nd "decreas ing cellular s ystem". Th e bi-d imensional cellular s ystems con sist of a regu lar g rid of cell s. Each cell takes different values, denoted by $a_{i,}$, and the system is up dated in di screte time st eps a ccording to s ome 
rule that depends on the value of sites in some neighbourhood around it. In this work, the neighbourhood structure is defined by the square region around a cell. The size of the grids, $\operatorname{Dim}_{x} x \operatorname{Dim}_{y}$, for the cellular systems is previously fixed. $\operatorname{Dim}_{x}$ (rows) is equal to the number of input ne urons N, plus the number of output ne urons M, and Dim (columns) corresponds with the maximum number of hidden neurons. In the next, the initial configurations, $\mathrm{p}$ ossible $\mathrm{v}$ alues of each cell a nd the ev olution ru les of the growing and decreasing cellular systems are presented.

\subsection{Growing Cellular System}

The growing cellular system is designed in order to obtain FNN architectures with a large number of connections between the input and hidden layer and between hidden and output layer. The initial configuration of the growing cellular system is given by $\mathrm{n}$ seeds, $\left(s_{1}, s_{2}, \ldots, s_{n}\right)$, called " growing se eds" ( GS). E ach se ed $\mathrm{i}$ s de fined by $\mathrm{t}$ wo coordinates which indicates the positions of the see $d$ in the grid. That positions are provided by the genetic algorithm module. In order to apply the automata rule the first time each seed is replicated over its quadratic neighbourhood, in such a way that if a new seed has to be placed in a position previously occupied by another seed, the first one is replaced. Thus, the value $a_{i j}$. of a cell can take two possible values: $a_{i j}=0$ when the cell is inactive and $a_{i j}=s_{\mathrm{k}}$ if the cell contains the seed $s_{k}$.

The $\mathrm{r}$ ule of $\mathrm{t}$ he gr owing $\mathrm{c}$ ellular $\mathrm{s}$ ystem ha $\mathrm{s}$ been de signed $\mathrm{t} \mathrm{o}$ allow the reproduction of growing seeds. The idea is to copy a particular growing seed $s_{k}$ when a cell is inactive a nd $t$ here are at leas $t$ th ree $i$ dentical $g$ rowing $s$ eeds $i \mathrm{n}$ its neighbourhood. The rule of the growing automata cellular is defined in equation 1:

$$
\begin{aligned}
& a_{i, j}^{(t+1)}=s_{k} \text { if } \begin{array}{l}
a_{i, j}^{(t)}=0 \mathrm{AN} \mathrm{D} \\
\\
a_{i-1, j-1}^{(t)}=a_{i-1, j}^{(t)}=a_{i-1, j+1}^{(t)}=s_{k} \text { OR } a_{i+11, j-1}^{(t)}=a_{i+1, j}^{(t)}=a_{i+1, j+1}^{(t)}=s_{k} \text { OR } \\
a_{i-1, j-1}^{(t)}=a_{i, j-1}^{(t)}=a_{i+1, j-1}^{(t)}=s_{k} \text { OR } a_{i-1, j+1}^{(t)}=a_{i, j+1}^{(t)}=a_{i+1, j+1}^{(t)}=s_{k} \text { OR } \\
a_{i-1, j-1}^{(t)}=a_{i-1, j}^{(t)}=a_{i, j-1}^{(t)}=s_{k} \text { OR } a_{i-1, j}^{(t)}=a_{i-1, j+1}^{(t)}=a_{i, j+1}^{(t)}=s_{k} \text { OR R } \\
a_{i, j-1}^{(t)}=a_{i+1, j-1}^{(t)}=a_{i+1, j}^{(t)}=s_{k} \text { OR } a_{i+1, j}^{(t)}=a_{i+1, j+1}^{(t)}=a_{i, j+1}^{(t)}=s_{k}
\end{array} \\
& a_{i, j}^{(t+1)}=a_{i, j}^{(t)} \text { in other case }
\end{aligned}
$$

According to that rule, a seed $s_{k}$ is reproduced when there are at least three identical growing seeds in its neighbourhood, which must be located in the same row, or in the same column or in the corner of the neighbourhood.

\subsection{Decreasing Cellular System}

Once the growing cellular system is expanded, most of the cell $\mathrm{s}$ in th e grid are occupied by growing s eeds. If th e presence of a growing seed is considered as the presence of a connection in the network, could be convenient to remove seeds in the grid in order to obtain a large variety of architectures. Hence, the decreasing cellular system is $\mathrm{i}$ ncorporated $\mathrm{t}$ o remo ve $\mathrm{c}$ onnections. Th e i nitial co nfiguration of th $\mathrm{e}$ decreasing cellular system is given by the final configuration of the growing cellular system and by m seeds $\left(d_{l}, \ldots d_{m}\right)$, called "decreasing seeds" (DS). Each seed is defined also by two coordinates and they are provided by the genetic algorithm module. The value $a_{i j}$ of a cell in this automata can be: $a_{i j}=0$ when the cell is inactive; $a_{i j}=s_{k}$ when the cell contains the growing seed $s_{k}$ and $a_{i j}=d_{r}$ if the cell contains the decreasing seed $d_{r}$. 
The rule of the decreasing cellular system is designed to remove growing seeds in the gr id. A gr owing seed $s_{k}$ is r emoved w hen t wo c ontiguous ne ighbouring cells contain i dentical growing se eds and a nother ne ighbouring cell c ontain a decreasing seed. The rule of the decreasing cellular system is defined as:

$$
\begin{gathered}
a_{i, j}^{(t+1)}=d_{r} \text { if } \quad\left(a_{i, j}^{(t)}=a_{i-1, j-1}^{(t)}=a_{i, j-1}^{(t)}=s_{k} \text { A N D } a_{i-1, j}^{(t)}=d_{r}\right) \mathrm{OR} \\
\left(a_{i, j}^{(t)}=a_{i-1, j-1}^{(t)}=a_{i-1, j}^{(t)}=s_{k} \mathrm{AND} a_{i, j-1}^{(t)}=d_{r}\right) \mathrm{OR} \\
\left(a_{i, j}^{(t)}=a_{i-1, j+1}^{(t)}=a_{i, j+1}^{(t)}=s_{k} \mathrm{AND} a_{i-1, j}^{(t)}=d_{r}\right) \mathrm{OR} \\
\left(a_{i, j}^{(t)}=a_{i-1, j}^{(t)}=a_{i-1, j+1}^{(t)}=s_{k} \mathrm{AND} a_{i, j+1}^{(t)}=d_{r}\right) \mathrm{OR} \\
\left(a_{i, j}^{(t)}=a_{i, j-1}^{(t)}=a_{i+1, j-1}^{(t)}=s_{k} \mathrm{AND} a_{i+1, j}^{(t)}=d_{r}\right) \mathrm{OR} \\
\left(a_{i, j}^{(t)}=a_{i, j+1}^{(t)}=a_{i+1, j+1}^{(t)}=s_{k} \mathrm{AND} a_{i+1, j}^{(t)}=d_{r}\right) \mathrm{OR} \\
a_{i, j}^{(t+1)}=a_{i, j}^{(t)} \text { in other case }
\end{gathered}
$$

Similar rules could be used, but the design must enforce that not all growing seed in the grid are removed.

\subsection{Evolving Growing and Decreasing Cellular System}

In or der to e volve a nd to combine both growing and decreasing cellular systems, a special procedure th at all ows th e $\mathrm{c}$ onvergence $\mathrm{t}$ oward a fi nal con figuration is proposed (see Fig 1):

1. All cells in the grid are set to the in active state and the growing seeds provided by the genetic module are located in the grid. The growing seeds are replicated over their quadratic neighbourhood.

2. The rule of the gr owing cellular s ystem is a pplied until no more rule conditions could be fired and a final configuration is reached.

3. The decreasing seeds are placed in the grid.

4. The rule of the decreasing cellular system is applied until the final configuration is reached.

5. A binary matrix $M$ is finally obtained, replacing the growing seeds by an 1 and the decreasing seeds or inactive cells by a 0 . That matrix will be used by the neural network module to obtain a FNN architecture.

\section{Experimental Results}

In this paper, the cellular approach has been tested for the parity problem. The fitness function provided to the genetic a lgorithm module is the i nverse of computational effort, equation 3 (a). Where " $\mathrm{c}$ " is the number of connections in the FNN architecture and " $t_{c}$ " the number of training cycles carried out. If th e network doesn't reach the defined error, it is trai ned a maximu m of cycles and the fit ness value associated is given by the equation $3(\mathrm{~b})$, where " $\mathrm{e}_{\text {re ached }}$ " is the error reached and " $\mathrm{e}_{\mathrm{fixed}}$ " the error previously fixed.

The parity problem is a mapp ing problem wh ere the do main s et co nsists of all distinct N-bit binary vectors and the results of the mapping indicates whether the sum of $\mathrm{N}$ components of the binary vector is odd o even. In most current studies (Sontag, 1992) shown that a sufficient number of hidden units for the network is $(\mathrm{N} / 2)+1$ if $\mathrm{N}$ is even and $(\mathrm{N}+1) / 2$ if $\mathrm{N}$ is odd. In this work parity seven has been considered as a 
study case. Hence, the network will have 7 input neurons and 1 output. Thus, the size of the grid would be $8 \times 64$.

The number of gr owing and decreasing se eds ha $\mathrm{s}$ been $\mathrm{m}$ odified a nd $\mathrm{t}$ he architectures ob tained with the cell ular app roach for the different number of seeds after 100 generations have four hidden neurons and most of them are fully connected. Only in one case (5-5), the architecture is not fully connected, the first input neurons is on ly con nected t $\mathrm{o}$ on e hidden n euron, wit hout $\mathrm{c}$ onnections t $\mathrm{o}$ the res $\mathrm{t}$ of hidden neurons. All of then obtain percentage of train and test errors around $90 \%$ and $80 \%$, respectively. When the direct en coding is used to find the optimal architecture, the length of $\mathrm{t}$ he $\mathrm{c}$ hromosome i s 343 ( 7inputs $\mathrm{x} 49$ hidden) and more complex architectures are ob tained. A fter 300 generations t he a rchitecture has 48 hidden neurons and $48 \%$ of connectivity.

For the g enerative capacity of the metho d $10000 \mathrm{ch}$ romosomes are ran domly generated, with $7 \mathrm{GS}$ and $7 \mathrm{DS}$. And the nets obtained, indicating how much hidden nodes and connections has each one, are shown in Fig 2. For a FNN, with " $H$ " hidden nodes, $\mathrm{N}$ inputs and $\mathrm{M}$ outputs there is a maximum $(H(N+M))$ and a mi nimum $(H)$ number of connections, and it is di splayed in $\mathrm{F}$ ig 2 . $\mathrm{T}$ he ne ts o btained c over the search space of FNN on the whole.

$$
F=\frac{1}{\left(c \cdot t_{c}\right)} \text { (a) } \quad F=\frac{1}{c \cdot t_{c}} \cdot\left(e_{\text {fixed }} / e_{\text {reached }}\right)(\mathrm{b})
$$

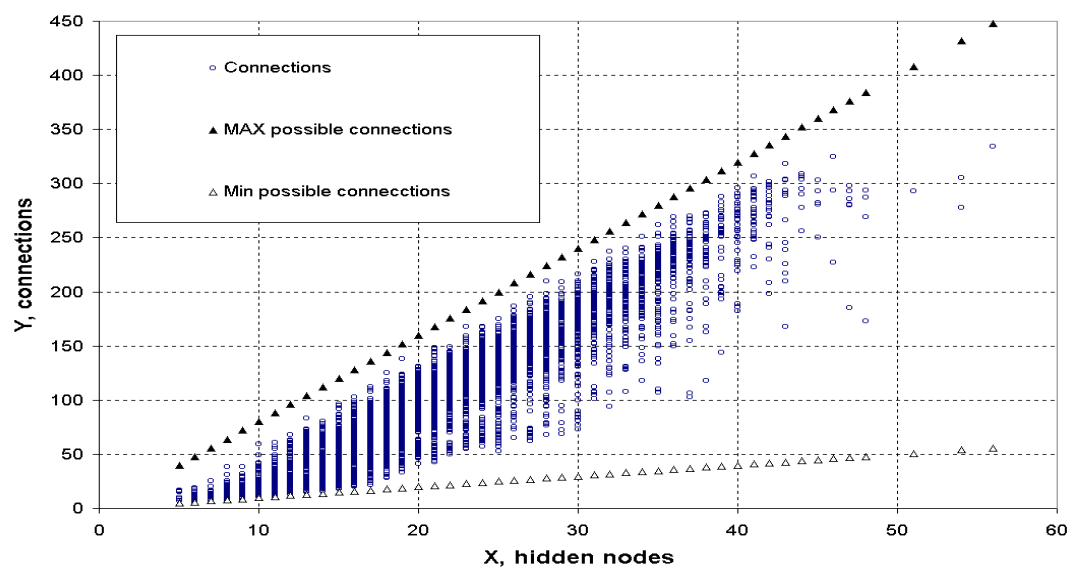

Fig. 2. Generative capacity for parity problem. A poi nt represents a $\mathrm{NN}$ wi th $\mathrm{X}$ hidden nodes and $\mathrm{Y}$ connections

\section{Conclusions and Future Work}

Cellular automata are $g$ ood cand idates $f$ or no n-direct cod ification's. Th e fi nal representation has a reduced si ze and could be $\mathrm{c}$ ontrolled by the $\mathrm{n}$ umber of se eds used. The results shown that the cellular scheme presented in this paper is able to find appropriate FNN a rchitectures, and the nets obtained a re independent of how many seeds (GS and DS) are placed in the CA. In addition, the number of generations over 
the population is less when the indirect encoding approach is used instead of direct codifications.

In future works not any individual in the population will have the same number of growing and decreasing seeds, i.e. a seed in the chromosome could be a growing seed or decreasing seed. Besides, s ome issues about Ne ural Network Module and fit ness function used, i.e. how punish the nets to increase the search, will be studied in future works.

\section{References}

[Alba 1993] Alba, E., Aldana, J. F., and T roya, J. M.: Fully automatic RNA design: a genetic approach. X. Yao: Evolutionary Artificial Neural Networks 45 In Proc. of Int'l Workshop on Artificial Neural Networks (IWRNA'93), p ages 3 99-404. S pringer-Verlag, 19 93. L ecture Notes in Computer Science, Vol. 686.

[Fogel et al, 1990] D.B. Fogel, Fogel L.J. and Porto V.W. Evolving Neural Network, Biological Cybernetics, 63, 487-493, 1990.

[Gruau, 1992] Gruau F. "Genetic Synthesis of Boolean Neural Networks with a Cell Rewriting Developmental P rocess". P roceedings of C OGANN-92 I nternational W orkshop o $\mathrm{n}$ Combinations of Ge netic Alg orithms an d Neural Networ ks, pp. 5 5-74, I EEE Comp uter Society Press, 1992.

[Gutierrez et. al., 200 1] G. Gut iérrez1, P. Isasi, J. M. M olina, A. Sanchís a nd I. M. Gal ván. Evolutionary $\mathrm{C}$ ellular $\mathrm{C}$ onfigurations $\mathrm{f}$ or Desi gning $\mathrm{F}$ eed-Forward Neur al Net works Architectures. Connectionist $\mathrm{M}$ odels of $\mathrm{n}$ eurons, $\mathrm{L}$ earning $\mathrm{P}$ rocesses and $\mathrm{Ar}$ tificial Intelligence. 6th International Work-Conference on Artific ial Ne ural Ne tworks, IWANN 2001. Proceedings, Part I. LNCS 2084. J. Mira, A Prieto (Eds.) Springer.

[Kitano, 1990] Kitano, H.: Designing Ne ural Networks using Genetic Algorithms with Graph Generation System, Complex Systems, 4, 461-476, 1990.

[Miller et al. , 1989] Geoffrey F. Miller, P eter M. Todd, and S hailesh U. Heg de. Desi gning Neural Networks using Genetic Algorithms. In J. David Schaffer, editor, Proceedings of the Third I nternational C onference on Gen etic Al gorithms, pa ges 379-384, San Mateo, California, 1989. Philips Laboratories, Morgan Kaufman Publishers, Inc.

[Molina e t a 1., 2 000] Mo lina, J . M., To rresano, A ., G alván, I. M., Is asi, P., Sa nchis, A.: Evolution of Context-free Grammars for Designing Optimal Neural Networks Architectures. GECCO 2000, Workshop on Evolutionary Computation in the Development of ANN. USA. Julio, 2000

[Sontag, 92] E. D. S ontag. F eedforward net s for interpolation and classification. Journal of Computer and System Sciences, 45, 1992.

[Wolfram, 1988] S. Wolfram. Theory and a pplications of cellular aut omata. W orld S cientific, Singapore, 1988.

[Yao, 1999] Y ao, X. 1999. Evolving artificial neural networks, Proceedings of the IEEE v ol. 87, no. 9, p.1423-1447. 